\title{
Algorithmic Computation of Annamalai's Geometric Series and Summability
}

\section{Chinnaraji Annamalai}

Vinod Gupta School of Management, Indian Institute of Technology, Kharagpur, India

\section{Email address:}

anna@iitkgp.ac.in

\section{To cite this article:}

Chinnaraji Annamalai. Algorithmic Computation of Annamalai's Geometric Series and Summability. Mathematics and Computer Science. Vol. 3, No. 5, 2018, pp. 100-101. doi: 10.11648/j.mcs.20180305.11

Received: October 12, 2018; Accepted: October 23, 2018; Published: October 24, 2018

Abstract: This paper presents a new mathematical technique for computation of geometric series and summability. This technique uses Annamalai's computing model of algorithmic geometric series and its mathematical structures for further development of the infinite geometric series and summability. This could be very interesting and informative for current students and researchers.

Keywords: Mathematics of Computation, Summability, Algorithmic Geometric Series

\section{Introduction}

Annamalai's computing model [1] has provided a novel approach for computation of geometric series in a new way.

$$
\begin{gathered}
\sum_{i=-m}^{n-1} a x^{i}=\frac{a\left(x^{n}-x^{-m}\right)}{x-1} \Leftrightarrow a x^{n}=a x^{n},(x \neq 1) \\
a \sum_{i=k}^{\alpha} x^{i}=\frac{a x^{k}}{1-x}(0<x<1)
\end{gathered}
$$

where $\boldsymbol{k}>0$ is an integer constant.

Algorithmic Geometric Series

$$
\begin{aligned}
& \text { (1a) } \sum_{i_{1}=0}^{\infty} \boldsymbol{a} \boldsymbol{x}^{i_{1}}=\frac{\boldsymbol{a}}{1-\boldsymbol{x}}(1 \boldsymbol{b}) \sum_{i_{1}=\boldsymbol{k}}^{\infty} \boldsymbol{a} \boldsymbol{x}^{i_{1}}=\frac{\boldsymbol{a} \boldsymbol{x}^{\boldsymbol{k}}}{1-\boldsymbol{x}} \\
& \text { (2a) } \sum_{i_{1}=0}^{\infty} \sum_{i_{2}=i_{1}}^{\infty} a x^{i_{2}}=\sum_{i_{2}=0}^{\infty} a x^{i_{2}}+\sum_{i_{2}=1}^{\infty} a x^{i_{2}}+\sum_{i_{2}=3}^{\infty} a x^{i_{2}}+\ldots . .=\frac{a}{(1-x)^{2}} \\
& \text { (2b) } \sum_{i_{1}=k}^{\infty} \sum_{i_{2}=i_{1}}^{\infty} a x^{i_{2}}=\sum_{i_{2}=k}^{\infty} a x^{i_{2}}+\sum_{i_{2}=k+1}^{\infty} a x^{i_{2}}+\sum_{i_{2}=k+2}^{\infty} a x^{i_{2}}+\ldots . .=\frac{a x^{k}}{(1-x)^{2}}
\end{aligned}
$$

Similarly, we can further generate the infinite geometric series and summability. 
$(N a) \sum_{i_{1}=0}^{\infty} \sum_{i_{2}=i_{1}}^{\infty} \sum_{i_{3}=i_{2}}^{\infty} \ldots \sum_{i_{n}=i_{n-1}}^{\infty} a x^{i_{n}}=\frac{a}{(1-x)^{n}}(N b) \sum_{i_{1}=k}^{\infty} \sum_{i_{2}=i_{1}}^{\infty} \sum_{i_{3}=i_{2}}^{\infty} \ldots \sum_{i_{n}=i_{n-1}}^{\infty} a x^{i_{n}}=\frac{a x^{k}}{(1-x)^{n}}$

Here, if $\boldsymbol{a}=1$, then

$$
\sum_{i_{1}=0}^{\infty} \sum_{i_{2}=i_{1}}^{\infty} \sum_{i_{3}=i_{2}}^{\infty} \ldots \sum_{i_{n}=i_{n-1}}^{\infty} x^{i_{n}}=\frac{1}{(1-x)^{n}} \& \sum_{i_{1}=k}^{\infty} \sum_{i_{2}=i_{1}}^{\infty} \sum_{i_{3}=i_{2}}^{\infty} \ldots \sum_{i_{n}=i_{n-1}}^{\infty} x^{i_{n}}=\frac{x^{k}}{(1-x)^{n}}
$$

Note that the above serial numbers denote the ranges $(1 \boldsymbol{a}),(2 \boldsymbol{a}),(3 \boldsymbol{a}), \ldots,(\mathrm{Na})$ and $(1 \boldsymbol{b}),(2 \boldsymbol{b}),(3 \boldsymbol{b}), \ldots,(\mathbf{N} \boldsymbol{b})$.

\section{Conclusion}

In the research study, a novel technique has been presented for formation and computation of infinite geometric series and summability.

\section{Reference}

[1] Annamalai C 2018 “Annamalai's Computing Model for Algorithmic Geometric series and Its Mathematical Structures”, Mathematics and Computer Science - Science Publishing Group, USA, Vol 3(1), pp 1-6.

\section{Biography}

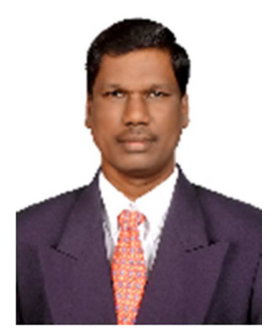

Chinnaraji Annamalai has experience for more than 20 years in research and teaching of computer science and information technology and admin. He has published several papers in diverse fields of science and technology. Also, he has reviewed recent IT and Computer books published by William Stallings (USA) and many other articles published by IEEE journals and top journals in the fields of computing sciences and its related subjects. Presently he is working at Indian Institute of Technology Kharagpur. 